\title{
L'inclusività nelle scuole, la società aperta e il suo medium
}

\author{
Danilo Marro
}

23 February 2018

\section{Breve introduzione}

Nella seguente trattazione s'individueranno, all'interno della cornice scolastica permeabile alle diverse dinamiche sociali che vengono imposte dall'attuale contesto storico, progettualità e progressi indirizzati verso un principio di inclusività. Partendo dall'analisi dei significati di inclusività, interculturalità e multiculturalità la descrizione si dispiegherà su più sub-oggetti concorrenti a delineare le qualità del pensiero interculturale, quest'ultimo tendente ad educare e a permettere l'individuo di essere solidale e interagente con la diversità delle culture.

Si analizzerà, inoltre, il fenomeno del cyberbullismo e il suo spazio, il suo medium.

\section{'Diversità', integrazione e inclusività}

Nell'attuale fase storico-sociale, la 'diversità' rappresenta per la scuola l'elemento culturale fondante della propria azione, articolata in modo competente in una pluralità di sostegni, da concretare mediante indispensabili sinergie al suo interno e cooperazioni solidali con la famiglia, il territorio e il terzo settore.

Nella messa a fuoco di tale azione significativa, la scuola si è confrontata con il progressivo cambiamento che ha caratterizzato la propria progettualità educativa e le prassi organizzativo-didattiche determinate dall'accesso alla scuola, per via 'normativa', di tutti degli allievi con disabilità tra gli anni Settanta e Novanta: dalla legge 118/1971, alla legge L.517/1977, alla Sentenza della Corte Costituzionale n.215/1987, alla legge n.104/1992 e al D.P.R del 1994.

Si tratta di norme che hanno anticipato e segnato, per certi versi, lo sviluppo della sensibilità pubblica attraverso passaggi che vanno dall'esclusione e dalla correlativa istituzionalizzazione separata, ossia «la tendenza a inserire gli alunni disabili in classi differenziali, che potevano avvalersi di assistenza medica, di strutture appositamente predisposte e di insegnanti specializzati», all'inserimento e all'integrazione, e infine all'inclusione e alla partecipazione. 
A fronte di ciò, tuttavia, è da ricordare che sono state anche le buone prassi sperimentate dalla scuola che hanno dato una forte spinta alle iniziative legislative stesse, incidendo in modo rilevante sul loro ingresso nel contesto scolastico e sociale.

In questo quadro dinamico si sono progressivamente sviluppati il dibattito e il tentativo di condivisione in primis del significato di integrazione e di inclusione e, più di recente, anche quello di partecipazione collegato ai bisogni educativi speciali (BES), sostenuti dalla ricerca scientifica più accreditata in materia e dall'ampia letteratura nazionale e internazionale. Su tali temi, esse hanno fornito un contributo rilevante all'arricchimento del quadro concettuale, pur nelle perduranti incertezze terminologiche e, talvolta, decisionali rispetto agli interventi più adeguati che ne dovrebbero conseguire.

Tuttavia, è fondamentale riconoscere che: «la prospettiva dell'integrazione, del riconoscimento dei diritti dei più deboli, del non ammettere la segregazione, l'umiliazione, la separazione, parte da una volontà [...] il punto di partenza rimane la volontà e [...] dove c’è volontà c'è un sentiero» e che "l'inclusione nella scuola è un aspetto dell'inclusione nella società più in generale".

La scuola e la società non sfuggono ad una filosofia implicita che, nella sua interventistica intrisa di una sorta di antropologia schematica e meccanicistica che si esprime soprattutto con un'esigenza di classificazione, prevale una visione dei bisogni come carenze da compensare in una logica assistenzialistica a favore del 'diverso' e delle categorie svantaggiate; una logica antitetica all'ottica dell'integrazione.

Le due essenziali riflessioni aiutano a rendere chiari i concetti-chiave della didattica inclusiva, che orientano la scuola nella concretizzazione dei diritti individuali, della partecipazione alla comunità, dell'uguaglianza di accesso e di successo, affinché non restino mere affermazioni di principio, ma siano riconducibili a principi-guida delle pratiche educative e del curricolo, tali da permettere che ogni allievo possa apprendere nel migliore dei modi e al massimo livello possibile.

Scaturisce da tali convinzioni che la rimozione delle barriere che ostacolano l'apprendimento e la partecipazione di ciascun alunno alla vita scolastica rappresenta il fondamento dell'educazione inclusiva, i cui principi ispiratori, radicati nella lotta alla discriminazione, alla diseguaglianza e all'esclusione dall'istruzione in particolare delle fasce più deboli, sono tesi ad orientare le politiche e le strategie socioculturali ed economiche dei Paesi per rendere davvero reale l'educazione per tutti. 
La dimensione scolastica inclusiva riguarda tutti gli alunni e va, quindi, correlata in modo significativo alla più ampia dimensione inclusiva dell'intera società, mettendo così in evidenza che i processi che vengono attivati costituiscono l'esito di un intreccio di competenze e responsabilità plurime che non possono essere a carico soltanto della scuola. Essi devono piuttosto convergere nelle sinergie reciproche tra quest'ultima e la comunità sociale, attraverso il perseguimento di fini comuni e la condivisione dei valori di giustizia, di equità, sussidiarietà e solidarietà, per promuovere davvero l'innalzamento della qualità dei traguardi formativi per tutti. Devono cioè cadere progressivamente le barriere che si frappongono all'apprendimento e alla partecipazione e far sì che la scuola si confronti dialetticamente con le proprie politiche organizzative e gestionali, rivedendo, se necessario, le proprie culture e prassi didattiche affinché possano corrispondere in modo sempre più efficace alle diversità in essa presenti. Si tratta dunque di continuare il cammino per l'integrazione verso l'inclusione che, come sappiamo, è segnato dall'incontro tra i linguaggi di frontiera della diversità, che hanno saputo indicare alla società civile la necessità di conoscere meglio, 'frequentandole', le instabilità, le precarietà inconsuete e spesso creative, le sensibilità 'differenti' e le nuove coscienze che hanno permesso di superare, sotto il profilo concettuale ed etico, i dualismi uguale/diverso, fermo/instabile, costante/incostante, per approdare in zone dell'essere e dell'esserci, quest'ultimo anche nel senso Heideggeriano di "apertura al mondo e comprensione di esso", più ricche e di maggiore consapevolezza.

\section{La cultura, subcultura e folclore}

L'incontro con l'Altro, con il 'diverso', rientra nelle situazioni della vita quotidiana; la coscienza e la comprensione di tale fenomeno in un contesto di "drammaticità del quotidiano" è una questione culturale e porta a formulare modelli e strategie etnocentriche ${ }^{2}$, non nuove nella storia dell'uomo, di assolutizzazione dei propri valori, di difesa, di salvaguardia della cultura e dell'identità del gruppo di appartenenza, a cui corrisponde l'attivazione di un meccanismo di negazione dell'altro basato sul concetto di diversità.

Dunque, in quest'ottica entra di merito nella trattazione, anche per chiarire in seguito il concetto di interculturalità, la questione di cultura e dei modelli culturali (complessi di idee, simboli e azioni) per 'comprendere' le culture Altre.

1 "drammaticità" nel senso di espressione degli eventi stocastici del quotidiano.

2 Etnocentrismo come "tendenza a giudicare la storia, la struttura sociale e la cultura dei gruppi umani diversi dal gruppo cui si appartiene secondo i valori propri di questo, tenuto come ideale centro e punto di riferimento dell'analisi". 
Nello studio antropologico-culturale la cultura, «totalità complessa che abbraccia nozioni, credenze, arti, costumi, abitudini e tutti gli altri tipi di capacità e di costanti attività, che sono propri dell'uomo in quanto membro della società» (E.B. Tylor 1871), viene analizzata e approfondita nella dinamica interna da cui sorge e nell'esteriorità in cui si concretizza, si struttura e si valorizza e l'uomo, attraverso essa e le sue forme interne che s'identificano come modelli culturali, avverte la necessità di spiegare, comprendere, dare un senso alla realtà e agli accadimenti della vita e, quindi, interpretarli. Qui, «I modelli culturali sono da intendersi come l'integrazione logica e coerente tra le rappresentazioni mentali e il bisogno della persona di interpretare e dare senso alla realtà e alla vita, e consentono alla persona umana di orientarsi nella ricerca di risposte atte a soddisfare tale bisogno».

Tuttavia, anche se l'appartenenza e l'identificazione con una cultura costituiscono un elemento importante per la formazione della personalità di base di un individuo, e i modelli culturali gli strumenti interpretativi della realtà, l'antropologia culturale deve indirizzare il suo contributo verso una condizione necessaria di relativismo culturale per lo studio e alla conoscenza delle diverse culture, le sottoculture e le culture subalterne. Altro non sono, queste ultime, che la manifestazione di una stratificazione culturale e sociale in cui la sottocultura, quale manifestazione specifica di un gruppo all'interno di una comunità più ampia, non possedendo una completa autonomia, e pur mantenendo importanti elementi di separazione e distinzione, parteciperebbe in qualche misura, alla cultura dominante.

Tuttavia, «l'antropologia non può considerare le espressioni culturali in termini di superiorità o di inferiorità, ma necessariamente e semplicemente, come culture differenti, a volte concorrenti, ma sempre espressione delle attività mentali dell'uomo in determinate coordinate spazio-temporali, degna di rispetto e di considerazione».

Pertanto, se col termine di cultura subalterna si rimanda a tutto ciò che comporta in riferimento ad una cultura egemone, con quello di folclore, secondo il pensiero gramsciano, si considera una piena autonomia e coerenza, espressione della mente dell'uomo e specifica visione della realtà e della società. Questa si colloca all'interno di una cultura complessa ufficialmente riconosciuta, e con questa capace di interagire, che include tutti gli aspetti della vita umana, offrendone gli strumenti interpretativi ed esplicativi, filtrati attraverso un quadro di riferimento valoriale.

Gramsci, fin dagli esordi del suo percorso intellettuale ritiene che il folclore non può essere considerato come una "bizzarria, una stranezza, una cosa ridicola", ma come "una cosa 
molto seria", proponendo di studiare il folclore come una diversa concezione del mondo, non ufficiale ma degna di considerazione e di rispetto. «Si può dire che finora il folclore sia stato studiato prevalentemente come elemento pittoresco. Occorrerebbe studiarlo invece come concezione del mondo e della vita», implicita in grande misura, di determinati strati (determinati nel tempo e nello spazio) della società, in contrapposizione (anch'essa per lo più implicita, meccanica, oggettiva) con le concezioni del mondo 'ufficiali'(o in senso più largo delle parti colte della società storicamente determinate) che si sono successe nello sviluppo storico. «Il contributo di Gramsci, anche se implicitamente offerto al pensiero antropologico, e l'attuale maturazione dell'antropologia culturale, invitano al superamento del semplice concetto di folclore come "cultura volgare" o "sub-cultura" riposizionando il folclore come “cultura-altra”, riflesso della pluralità dei modi di essere dei vari gruppi sociali, che non implica antagonismo né subalternità, né conflitto, ma si situa concretamente nella linea delle realtà sostanzialmente parallele. Di fatto, il folclore, come modello culturale, è espressione di un sano pluralismo che presuppone la disponibilità interiore al dialogo, all'interscambio e all'arricchimento vicendevole».

Nel 1949 Ernesto De Martino pubblica un saggio dal titolo "Intorno a una storia del mondo popolare subalterno", nel quale dichiara la sua apertura alle problematiche meridionalistiche.

Con quest'opera inaugura quella che è stata definita dagli studiosi di antropologia culturale antropologia del negativo, l'antropologia delle masse che non "fanno storia" in senso crociano, ma che "irrompono nella storia". "Questo tema, di grande suggestione antropologica, provocò sia la reazione degli storicisti di matrice idealista, i quali affermavano che il primitivo, le plebi non potevano essere oggetto di storia; sia la reazione di marxisti che vedevano nelle masse popolari un potenziale proletariato da riscattare con l'affermazione della coscienza di classe. Inoltre, lo studio dei fenomeni magico-religiosi, aprì, in De Martino, la prospettiva di una ulteriore riflessione sui rapporti o "incontro" tra l'etnologo e l'oggetto della conoscenza, ossia gli individui e le comunità studiate».

È questa la teoria definita come "umanesimo etnografico" o "etnocentrismo critico": l'antropologo deve partire dalla considerazione che, essendo forte il radicamento nella tradizione storica e intellettuale occidentale, e greco-romana-rinascimentale, egli non può non essere "etnocentrico", cioè non può non partire da una base storico-conoscitiva che non può 
essere facilmente eliminata, né è opportuno farlo ${ }^{3}$. Lo sguardo che gettiamo sul mondo delle diversità culturali è dunque orientato, governato da categorie ed esperienze storiche ineliminabili, ed apprezzabili.

Pur sostenendo l'impossibilità di uscire dalle proprie categorie logiche e culturali lasciandosi andare a una concezione 'difensivistica' dell'Occidente, De Martino si applica nella ricerca di un Altro modo di interpretare e ridefinire le culture che prescinde dal netto dualismo etnocentrismo/relativismo.

Dunque, l'etnocentrismo critico rappresenta l'impegno da parte dell'antropologo di fronte ad una cultura 'altra' di presa di coscienza critica dei limiti della propria storia culturale, politica e sociale; egli non può comprendere il diverso se non partendo, prima, dalle categorie conoscitive della propria cultura. Tale sforzo conoscitivo tende a produrre un esame critico delle categorie usate dall'Occidente per conoscere l'altro e un loro ampliamento.

Non è da sottovalutare, quindi, il prezioso contributo che Ernesto De Martino potrebbe fornire nell'ambito della ricerca pedagogica interculturale, con particolare riferimento alle ricerche pedagogiche nei contesti educativi eterogenei.

Nella sua opera, già citata in precedenza, dove viene suggerito come alcune delle riflessioni che portavano De Martino a criticare gli approcci naturalistici e destoricizzati alle culture rurali del Sud Italia, potrebbero essere preziose, anche oggi, per la ricerca pedagogica interculturale al fine di ridurre 'quei rischi' di un approccio solo culturalista alla complessità emergente negli odierni contesti educativi. In secondo luogo, dove sussiste il pensiero di una crisi culturale, dall'opera postuma "La fine del mondo", per cui le culture potrebbero essere analizzate come forme di "progettazione comunitaria" attraverso le quali le persone possono essere "attivamente presenti" nel/al mondo, la ricerca pedagogica interculturale odierna riuscirebbe a ricontestualizzare l'obiettivo di promuovere l'interazione fra componenti migranti, postmigranti ed autoctone della popolazione nella forma di un processo di tipo comunitario di quotidiana e permanente ricostruzione culturale all'interno di contesti eterogenei come quelli contemporanei?

\section{L'interculturalità e gli obiettivi per una scuola interculturale}

3 La posizione di De Martino nasce da una polemica vivace nei confronti di un atteggiamento mentale e di una teoria antropologica che egli considera "opposta" all'etnocentrismo critico: il relativismo culturale, che risale all'antropologo americano Melville Herskovits. 
Prima di addentrarci nella trattazione dell'interculturalità e della sua pedagogia conviene conviene preliminarmente chiarire il concetto di multiculturalità.

Si tratta di una categoria descrittiva, un fatto oggettivo, con la quale si può indicare «il vivere su uno stesso territorio di popolazioni che differiscono per provenienza geografica, culturale e lingua madre e che non veicola in sé giudizi di valore né tantomeno analisi, bensì fornisce il semplice quadro di tali comunità senza che esse a priori debbano interagire fra loro, confrontandosi o avendo degli scambi». Una prospettiva statica, dunque, che non prevede necessariamente contatti fra i vari attori, lasciandoli sullo sfondo come universi paralleli.

L'interculturalità è l' intenzione di entrare nel mondo Altro; è una dimensione pedagogica, una risposta educativa e deve avere riscontro nell'intenzionalità.

Secondo il documento ministeriale del 2007, parte degli interventi concreti finalizzati a rendere operante la logica della pedagogia inclusiva nella scuola sono, appunto, quegli interventi di integrazione ed interazione interculturale.

Essenzialmente, quest'ultima punta «alla creazione di un clima plurale che valorizzi la diversità nella pari dignità e ad una gestione pedagogica e didattica che favorisca l'instaurarsi di relazioni positive, contrastando i pregiudizi, il razzismo e la discriminazione».

Per far ciò, non si può che dare attenzione alla formazione di un significante progetto educativo, complementare a ciò che viene considerata "pedagogia generale" e che deve rendere consapevole l'individuo della molteplicità di identità possibili: la pedagogia interculturale.

Obiettivo primario del sistema interculturale è quello per poter attuare un "decentramento dallo sguardo", tale, cioè, da consentire aperture e possibilità di relazione non contemplate in precedenza. Secondo il principio del decentramento l'io, dislocandosi, arricchisce la propria identità rendendola plurale in virtù dell'ascolto, dell'accoglienza, della contaminazione e della narrazione dell'altro. In questo modo, decentrandoci, possiamo imparare chi siamo veramente attraverso lo sguardo dell'altro: nel contatto con ciò che è Altro si è in grado di costruire la propria immagine, attraverso processi di identificazione e differenziazione.

Si può, quindi, con il decentramento dallo sguardo, attuare il passaggio da una prospettiva etnocentrica ad una etnorelativa. 
Ulteriori obiettivi indicati in letteratura sono i seguenti:

- «tradurre nella pratica scolastica i principi - basati sulla Costituzione - del diritto allo studio e dell'eguaglianza scolastica: occorre prevenire o decostruire un clima di discriminazione, esclusione, pregiudizio, che, tra l'altro, crea un contesto sfavorevole all'apprendimento»;

- "educare alla cittadinanza partecipativa e non violenta, al rispetto dei diritti di tutti”;

- "favorire lo sviluppo di abilità relazionali e dialogiche che portino a confrontarsi con la diversità".

Per quanto riguarda le proposte didattiche, alcune elaborate in funzione di questi obiettivi hanno privilegiato lo sviluppo di competenze di tipo cognitivo volte alla maturazione di una forma mentis interculturale da parte dell'individuo.

Infatti, nel suo "Pedagogia dell'intercultura" F. Cambri chiarifica che «L'intercultura, però, è soprattutto la formazione di una nuova forma mentis, post-etnocentrica, dialogica, aperta all'ascolto e all'incontro, destrutturata rispetto ai suoi pregiudizi, rivolta al meticciamento visto come una risorsa».

G. Favaro e A. Boschetti insistono sulla necessità di una forma mentis che non deve riguardare solo la sfera cognitiva, ma deve essere favorita dall'acquisizione di competenze di tipo affettivo: «L'aspetto emotivo si rivela tanto più necessario in un contesto educativo come quello odierno, che ci pone nella condizione di accettare i conflitti presenti nelle nostre scuole (e non di negarli!) e di affrontarli attraverso la costruzione di nuovi processi di negoziazione, complicati dalle difficoltà linguistiche, ma soprattutto dalle resistenze culturali di molti, che genericamente chiamiamo pregiudizi».

Si insiste, dunque, sul concetto di empatia definito come un concetto intenzionale che pone l'individuo «in ascolto dell'Altro» e nella condizione «di ritrovare nella propria storia dei momenti, degli episodi che ognuno ha vissuto e ha provato quando si è sentito straniero a se stesso»

M. Sclavi, invece, per lo sviluppo del decentramento cognitivo e la soluzione creativa dei conflitti, introduce un metodo ludico-umoristico basato su diversi consigli. 


\section{“Il medium è il messaggio”}

Uno sforzo importante da produrre nel raggiungimento di una formazione interculturale riguarda la costruzione di un'immagine della diversità culturale che sia alternativa rispetto agli stereotipi che quotidianamente i mass media, o in generale i media, contribuiscono a creare.

Prendendo in prestito l'espressione "il medium è il messaggio" da "Understanding media" del sociologo Marshall McLuhan, si vuole indicare, qui, che, al di là del contenuto specifico veicolato dai mass media, i criteri strutturali con cui viene organizzata la comunicazione da questi ultimi produce un'influenza sui destinatari del messaggio; produce effetti pervasivi sull'immaginario collettivo. L'autore, interpretando il suo concetto, si accosta a Sergej Michajlovič Ejzenstejn associando la sua teoria a quella cinematografica del montaggio delle attrazioni: «un accostamento produttivo tra materiali di diversa origine che fa nascere pensieri nuovi da materiali preesistenti, e segue la orma associativa propria del pensiero umano».

Invece, gli effetti 'mediali' che presuppongono cambiamenti che avvengono a livello di ambiente sociale e culturale sono più difficili a vedersi, sia perché agiscono sulle strutture profonde della sfera cognitiva e sensoriale umana, cioè sul modo di pensare e sentire, e dunque sul modo in cui di fatto agiamo, sia perché tendiamo a darli per scontati; diamo cioè per scontato l'ambiente che ci sta attorno: «nelle ere della meccanica, avevamo operato un'estensione del nostro corpo in senso spaziale. Oggi, dopo oltre un secolo di impiego tecnologico dell'elettricità, abbiamo esteso il nostro stesso sistema nervoso centrale in un abbraccio globale che, almeno per quanto concerne il nostro pianeta, abolisce tanto il tempo quanto lo spazio».

\section{Cyberbullismo e cyberspazio: una rivoluzione comunicativa}

Sulla base di quanto trattato riguardo ai media e ai loro effetti sull'ambiente, sullo spazio e il tempo ridotti e sull'immaginario collettivo, si ritiene necessario esporre ciò che invece è creduta come "la piaga socialvirtuale dei nostri tempi".

I nuovi mezzi di comunicazione rendono tutto più veloce e diretto, superano i vecchi confini 
di spazio e tempo, consentono di portare delle relazioni al di là della realtà fisica e della corporeità. Evolvono continuamente, quindi forniscono sempre nuovi stimoli: dalla possibilità di parlare senza vedersi, allo scriversi (chat), allo scambio di foto o informazioni, in pubblico o in privato, a whatsapp; è un continuo stimolo, che tende a privilegiare lo strumento tecnologico come intermediario della relazione, come strumento efficace e sostitutivo della comunicazione corporea; un mezzo che frammenta la realtà in una molteplicità di stimoli dislocati nello spazio e nel tempo.

Tra i rischi gravi connessi ai mezzi di comunicazione diventa sempre più presente e violento il fenomeno del cyberbullismo. Strettamente correlato al bullismo, il cyberbullismo è abbastanza recente e i suoi effetti vengono a riscontrarsi nella realtà virtuale, nella dimensione immateriale: il cyberspazio.

Sebbene questo luogo non debba essere confuso con la rete Internet vera e propria, il termine è spesso usato per riferirsi ad «oggetti ed identità che esistono ampiamente all'interno della stessa comunicazione dei network, cosicché un sito web per esempio si potrebbe dire metaforicamente che esiste nel cyberspazio».

Il termine è stato coniato negli anni ' 80 dello scorso secolo dallo scrittore William Gibson nel romanzo "Neuromante", dove viene descritto come «un'allucinazione vissuta consensualmente ogni giorno da miliardi di operatori legali, in ogni nazione, da bambini a cui vengono insegnati i concetti matematici... una rappresentazione grafica di dati ricavati dai banchi di ogni computer del sistema umano. Impensabile complessità. Linee di luce allineate nel non-spazio della mente, ammassi e costellazioni di dati. Come le luci di una città, che si allontanano» (Gibson, 1984);

Ma, l'aspetto essenziale è sicuramente la facilità d'interazione fra gli utenti che ci navigano e che lo alimentano: nel momento in cui un'informazione pubblica si trova nel cyberspazio è virtualmente ed immediatamente a disposizione, comunità disperse possono pertanto comunicare per mezzo della condivisione di una telememoria sulla quale ogni membro legge e scrive. E' chiaro, dunque, che la funzione di messaggistica è ciò che regge virtualmente tale spazio, e non soltanto;

«un solo episodio, divulgato a migliaia di astanti, ad esempio la pubblicazione di un video su YouTube, può arrecare un potenziale danno alla vittima anche senza la sua ripetizione nel tempo; il video è sempre disponibile, può essere visto da migliaia di persone in tempi diversi. Lo stesso contenuto offensivo divulgato da un bullo può essere diffuso a cascata tra i 
riceventi, eventualmente anche non implicati nella relazione bullo-vittima. Non è quindi necessario, che l'atto offensivo venga ripetuto dallo stesso aggressore nel tempo. Una vasta platea di spettatori potrà comunque amplificare l'effetto dell'aggressione, con risultati devastanti per la vittima».

Di conseguenza, anche la possibilità di condividere è lo strumento che più favorisce tale fenomeno.

In generale possiamo, quindi, dire che il cyberbullismo, «quella forma di prevaricazione volontaria e ripetuta nel tempo, attuata mediante uno strumento elettronico, perpetuata contro un singolo o un gruppo con l'obiettivo di ferire e mettere a disagio la vittima di tale comportamento, che non riesce a difendersi» è un fenomeno nuovo che nasce proprio dalla diffusione delle nuove tecnologie e che risponde alle seguenti caratteristiche:

- E' volontario: cioè frutto di un comportamento deliberato, non accidentale o casuale; il cyberbullo sceglie ed è consapevole di perpetuare il suo comportamento deviante;

- Ripetuto: questo tipo di bullismo rispecchia un modello di comportamento che non è incidentalmente isolato, ma si ripete per lunghi periodi. Non è mai un episodio singolo;

- Arreca un danno: la vittima deve percepire che il danno è stato inflitto;

- Prevede l'uso di dispositivi elettronici: in modo non funzionale e sano, ma patologico e deviante;

- Pervasivo: il cyberbullismo può avvenire in qualsiasi momento e in qualsiasi luogo. Gli attacchi di cyber hanno insiti in sé un rischio maggiore, dovuto alla diffusione massiccia e istantanea che coinvolge un largo pubblico senza nessun tipo di controllo;

- Persistente: i contenuti diffusi in rete rischiano di rimanere online per lungo tempo e sono difficili da rimuovere, anche quando gli atti di bullismo cessano;

- È contraddistinto da anonimato e mancanza di empatia: la rete garantisce in molti casi l'anonimato. La distanza fisica creata dallo schermo riduce l'empatia, e quindi la capacità di comprendere lo stato d'animo della 'vittima';

Per la vittima è difficile risalire al molestatore ed ancora più difficile potrebbe essere reperirlo. Tuttavia, ogni comunicazione elettronica lascia delle tracce e l'anonimato è 
meramente illusorio.

L'anonimato del bullo associato alla possibilità di essere "un'altra persona" on-line sono in grado di indebolire gli impedimenti morali. È stato, infatti, constatato che, nel mondo virtuale, la gente osa, ovvero fa e dice cose che non farebbe o direbbe nella vita reale.

Per quanto riguarda l'assenza di limiti spazio-temporali, il cyberbullismo, a differenza del bullismo che si manifesta frequentemente in luoghi e momenti specifici (ad esempio, nel contesto scolastico), investe la vittima ogni volta che si collega al mezzo elettronico utilizzato dal cyberbullo;

Inquadrando più attentamente la figura dei cyberbulli,invece, questi «sono quei soggetti che non hanno il coraggio di mettersi di fronte alla vittima, infatti, per alcuni soggetti è molto più semplice essere nascosti, mantenere la propria invisibilità garantendosi così, l'opportunità (a loro avviso) di rimanere 'intoccabili' (fino un certo punto) e di colpire la vittima senza essere né visti e né scoperti» «Il cyberbullo, così come nel tradizionale bullismo, è indice di disagio e crisi di identità. Il giovane sente di esistere solo se viene notato con commenti, tag, post, per le azioni che ha realizzato ed usa internet perchè permette l'accesso ad un'ampia e numerosa platea alla quale raccontare e mostrare le proprie gesta» (Pisani, 2011).

Altro fenomeno che può derivare da un utilizzo non opportuno del mezzo di comunicazione è quello dello stalking. Questo è un termine utilizzato per indicare una serie di atteggiamenti tenuti da un individuo, detto stalker, che affliggono un'altra persona, perseguitandola, generandole stati di paura e ansia, arrivando persino a compromettere lo svolgimento della normale vita quotidiana e che, purtroppo, nel nostro paese ha avuto un percorso esteso e travagliato prima di essere stato riconosciuto come reato a tutti gli effetti.

\section{Bibliografia}

1. Comerci, N. (2008). La deiscenza dell'altro: intersoggettività e comunità in MerleauPonty. Mimesis Edizioni.

2. McLuhan, M. (1994). Understanding media: The extensions of man. MIT Press.

3. Pekerti, A. A., Vuong, Q. H., \& Napier, N. K. (2017). Double edge experiences of expatriate acculturation. Journal of Global Mobility, 5(3), 225-250. 
4. Moduli 3, 4 e 5 dagli insegnamenti su piattaforma telematica. 